\title{
Robustesse et canalisation : vision de généticiens
}

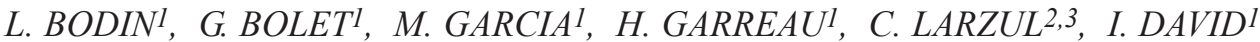 \\ ${ }^{1}$ INRA, UR631 Station d'Amélioration Génétique des Animaux, F-31320 Castanet-Tolosan, France \\ 2 INRA, UMR1313 Génétique Animale et Biologie Intégrative, F-78352 Jouy-en-Josas, France \\ ${ }^{3}$ AgroParisTech, Génétique Animale et Biologie Intégrative, 16 rue Claude Bernard, F-75231 Paris, France
}

Courriel :Loys.Bodin@toulouse.inra.fr

Bien qu'employé fréquemment dans le domaine des productions animales, le terme de robustesse n'a pas une définition standard et recouvre des concepts qui peuvent être différents selon les disciplines. Pour les généticiens, ce peut être la propriété d'un caractère exprimé de façon peu variable dans divers environnements. Cet article présente les hypothèses génétiques sousjacentes à ce dernier concept et les modèles associés d'interaction entre le génotype et le milieu.

Les objectifs de sélection des animaux domestiques s'adaptent constamment aux grandes évolutions de la production, du marché et de la société. Au démarrage des schémas modernes d'amélioration génétique (dans les années 60), ces objectifs ont été orientés vers l'augmentation des productions. Au cours de la décennie suivante, des objectifs de qualité des produits sont apparus, puis dans les années 90 des caractères «fonctionnels» (qualité des aplombs, morphologie mammaire...) ont été pris en compte pour obtenir des animaux mieux adaptés à leur contexte de production. A ce jour, beaucoup de populations souhaitent intégrer des objectifs non marchands qui doivent satisfaire à des contraintes ou besoins «environnementaux», «sociétaux», de «bien-être animal», etc.

Parmi ces nouveaux caractères, plusieurs concernent la relation de l'animal à son milieu. Les notions de robustesse, flexibilité, plasticité, rusticité, ou d'adaptation à des milieux difficiles sont souvent avancées comme nouveaux objectifs de sélection des populations animales avec des acceptions très variables selon les situations. L'imprécision de ces termes et les difficultés qu'elle entraîne proviennent du fait qu'ils appartiennent à trois terminologies différentes : celle de la vie courante (ex : rustique et rusticité), celle utilisée pour caractériser le monde du vivant et notamment les espèces animales sauvages, et finalement la terminologie spécifique des productions animales et de la génétique. Ce thème est si difficile à aborder que Debat et David (2001) ont écrit un article spécifique sur la définition historique de ces concepts, ainsi que de leur sens actuel et des sources de confusion possibles.

\section{1 / Concepts et définitions générales}

\section{1 / Le concept d'interaction} génotype $\times$ milieu au sens large

Lorsque l'effet d'une variation environnementale sur un phénotype est différent en fonction du génotype, il y a au sens statistique du terme une interaction entre les génotypes et les milieux. La valeur phénotypique $(\mathrm{P})$ d'un individu n'est plus alors simplement la somme des effets génétiques $(G)$ et environnementaux (E) soit $P=G+E$, mais elle inclut aussi un terme d'interaction $\left(\mathrm{I}_{\mathrm{GE}}\right)$, soit $\mathrm{P}=\mathrm{G}+\mathrm{E}+\mathrm{I}_{\mathrm{GE}}$. Cela ajoute une source de variance supplémentaire à la variance phénotypique $\left(V_{P}\right)$, égale à $V_{G}$ $+\mathrm{V}_{\mathrm{E}}$ dans le premier cas, à $\mathrm{V}_{\mathrm{G}}+\mathrm{V}_{\mathrm{E}}$ $+\mathrm{V}_{\mathrm{I}}$ dans le second. Ce concept d'interaction entre génotype et environnement $(\mathrm{G} \times \mathrm{E})$ a été très largement étudié, surtout dans le monde de l'amélioration des plantes (Freeman 1973).

L'hypothèse biologique la plus simple pour expliquer les interactions $\mathrm{G} \times \mathrm{E}$ est que les gènes qui contrôlent un caractère sont partiellement différents selon les milieux. Mais un concept plus large des interactions $\mathrm{G} \times \mathrm{E}$ fait intervenir d'autres hypothèses génétiques comme l'existence de gènes qui contrôlent la sensibilité aux variations environnementales.

La robustesse, définie comme la sensibilité aux variations du milieu dans lequel est exprimé un phénotype, fait partie de ce concept élargi. Nous allons focaliser cet article sur ce concept et sur les possibilités de sélection de la robustesse des caractères. Nous verrons que différentes hypothèses génétiques peuvent expliquer ce phénomène (encadré 1) et que plusieurs modèles mathématiques y sont associés (encadré 2).

\section{2 / Le concept de robustesse}

Le concept de robustesse est employé dans plusieurs disciplines et possède plusieurs définitions :

- en statistique, la robustesse d'un estimateur est sa capacité à ne pas être modifié par un petit changement dans les données ou dans les paramètres du modèle choisi pour son estimation ;

- dans le monde du vivant, la robustesse d'une population sauvage traduit sa capacité de survie (ou de maintien de l'espèce) dans une grande variété d'environnements. Le sanglier et le loup sont, à ce titre, plus robustes que le koala qui ne mange que des feuilles de quelques espèces d'eucalyptus ;

- en production animale, il existe au moins deux écoles pour définir la robustesse, selon qu'on l'associe à un potentiel de production globale ou à un potentiel de production spécifique.

Robustesse ou maintien d'un potentiel de production globale

Selon certains auteurs, la robustesse correspond à la capacité de survie de l'animal et au maintien d'un potentiel global de production. La robustesse peut ainsi être définie comme «l'aptitude à combiner un haut potentiel de production avec la capacité à endurer des stress, ce qui permet l'expression d'un haut potentiel de production dans une grande variété de conditions environnementales» (Knap 2005). Il est alors possible de définir un critère de robustesse comme la longévité dont 
la prise en compte dans un schéma de sélection ne diffère pas de celle des caractères de production classiques.

Du point de vue biologique, cette approche considère la robustesse comme la réponse non spécifique d'un animal aux stimulations du milieu, généralement considérées comme négatives et stressantes. La robustesse dépend donc du fonctionnement de l'axe corticotrope qui est, selon cette école, l'élément essentiel et privilégié du contrôle de la réponse au stress. De nombreuses expériences mettent en évidence la relation entre le fonctionnement de l'axe corticotrope et des caractères associés à la robustesse comme la résistance au stress, la survie ou la résistance aux maladies (Minozzi et al 2008). Il a, par exemple, été montré une association entre l'activité de l'axe adrénocortical hypothalamo-hypophysaire des rats et leur résistance au stress thermique (Michel et al 2007) et une relation entre le niveau de cortisol des fœtus porcins et leur valeur génétique pour la survie jusqu'au sevrage (Leenhouwers et al 2002). Parallèlement, l'activité de l'axe corticotrope intervient également sur le niveau de production. Par exemple, Foury et al (2007) ont établi une covariation du niveau de cortisol et de la composition de la carcasse des porcs entre et intra-lignées. Il est donc tout à fait envisageable d'améliorer la tolérance au stress lié aux conditions d'élevage pour diminuer la sensibilité des productions aux variations de facteurs environnementaux. De ce fait, plusieurs expériences de sélection sur l'activité corticotrope en réponse à un stress ont été conduites chez le poulet, la dinde, le porc (Brown et Nestor 1973, Gross et Siegel 1985, Foury et al 2007), mais le lien entre cette réponse et la stabilité des productions dans différents environnements n'est pas clairement établi. Chez la truite, une sélection divergente sur la tolérance au stress a été entreprise au travers de mesures d'élévation de cortisol plasmatique induite par une situation de confinement. Elle conduit à des différences significatives de croissance chez les parents mais pas chez les produits (Pottinger et Carrick 2000).

Cependant, si le rôle des facteurs de stress au travers de l'activité de l'axe corticotrope est indéniable pour les productions, ce n'est probablement pas le seul mécanisme biologique par lequel les facteurs environnementaux modulent l'expression des caractères. L'axe corticotrope ne contrôle en fait qu'une fraction de la part non spécifique de la robustesse.
Robustesse ou maintien d'un potentiel de production spécifique : relation avec la canalisation

Dès 1940, Waddington crée le concept de canalisation pour décrire l'augmentation de la robustesse d'un phénotype vis-à-vis des perturbations génétiques ou environnementales. Il distingue la canalisation génétique, qui regroupe les processus qui tamponnent les effets des mutations alléliques, et la canalisation environnementale, qui regroupe les processus tamponnant les effets de l'environnement (Waddington 1940). Ce concept est lié à celui de la plasticité qui considère l'ajustement des phénotypes en fonction de l'environnement et à celui de la stabilité du développement qui s'intéresse aux mécanismes qui maintiennent un phénotype constant malgré de petites variations aléatoires du développement. Telle que la définit cet auteur, la robustesse est la capacité à maintenir une production spécifique à un niveau relativement stable dans des environnements variés. Contrairement à l'approche précédemment décrite qui concevait la robustesse comme le maintien d'une production globale, cette seconde approche est attachée à un phénotype particulier et, par extension, à une production particulière.

Pour le généticien quantitatif, cela signifie le maintien de l'expression d'un caractère donné (ex : production de lait, production d'œufs, croissance...) de façon stable et peu dépendante des conditions environnementales. La robustesse n'est plus alors un caractère en tant que tel, plus ou moins lié aux caractères de production, mais est attachée à un caractère de production donné. Nous verrons plus loin que plusieurs théories génétiques expliquent cette plus ou moins grande stabilité des génotypes dans les différents milieux.

Bien que Waddington (1940) ait associé étroitement les termes de canalisation et de robustesse, on peut à l'heure actuelle définir plus spécifiquement la canalisation comme l'action de sélectionner pour diminuer la sensibilité aux variations environnementales et entraîner la moyenne du caractère vers une certaine valeur. Même si différents critères de sélection associant ces deux caractéristiques ont été développés (San Cristobal-Gaudy et al 1998a), la façon de les combiner pose un certain nombre de problèmes qui ne sont pas totalement résolus comme, par exemple, l'optimisation sur plusieurs générations des pondérations à attribuer à la sélection sur la moyenne et à la sélection sur la variabilité.

\section{2 / Intérêt de la canalisation des caractères pour l'amé- lioration génétique}

De nombreux arguments militent en faveur d'une prise en compte de la canalisation de certains caractères dans les schémas actuels d'amélioration génétique.

- La sélection visant à augmenter la moyenne d'un caractère n'est plus toujours l'objectif unique

L'augmentation de la moyenne engendrée par la réponse à la sélection ou par des améliorations des techniques d'élevage, aboutit parfois à un niveau optimum du point de vue biologique ou économique. C'est par exemple le cas de la prolificité de certaines races ovines qui présente un optimum variable selon les systèmes et conditions de production. Pour la race Lacaune, cet optimum est voisin de deux agneaux par portée ; audelà, le faible poids de naissance des jeunes, leur vitesse de croissance inférieure et leur plus grande mortalité ne compensent pas leur plus grand nombre. L'optimum étant atteint, les éleveurs peuvent chercher à homogénéiser au maximum les tailles de portée. On a ainsi montré que des naissances de deux agneaux par portée permettent d'améliorer le résultat d'exploitation de 30 à $35 \%$ par rapport à des situations où le même niveau de prolificité est obtenu par une portée simple et une portée triple (Benoit, communication personnelle). De très nombreux autres caractères comme le poids de carcasse des poulets de chair, le poids des foies gras de canard, le poids adulte des escargots, ainsi que l'aptitude à la dominance sociale ou l'intensité des réactions de peur chez la volaille, présentent aussi un optimum intermédiaire vers lequel il convient de canaliser la production.

\section{- Agriculture durable}

La mise en place d'une agriculture durable doit mobiliser des systèmes de production dans lesquels le milieu est moins contraint et contrôlé. De ce fait, ils induisent potentiellement des productions plus variables parce que générées dans des environnements partiellement moins stables. Pour conserver une production homogène, il convient de trouver de nouveaux moyens capables d'assurer la stabilité des productions malgré le relâchement des contraintes sur l'environnement. L'amélioration génétique, qui est une technique assez respectueuse des impératifs du développement durable, a un nouveau rôle important à jouer dans ce contexte. Un de ses nouveaux défis est donc de transférer la maîtrise des effets de 
l'environnement par le système de production à leur maîtrise par l'animal luimême.

\section{duits \\ - Amélioration de la qualité des pro-}

Dans de nombreuses situations, l'homogénéité des produits est un facteur de qualité en ce sens qu'elle permet, comme le demande l'industrie ou le marché, leur classification et leur assignation à des classes précises. Par exemple, les carcasses des poulets trop lourds ou trop légers risquent d'être abîmées lors de l'abattage. Le tri des produits (en fonction de leur taille, poids...) peut être fait par le secteur commercial, mais il est coûteux et il est plus intéressant que les productions soient naturellement homogènes.

- Globalisation et accroissement des échanges génétiques

Dans un contexte de globalisation et d'extension des milieux de production, les meilleurs reproducteurs sont ceux qui assurent une production élevée dans tous les environnements. Même si la combinaison optimum (qui n'est pas forcément économique, mais qui peut être aussi sociétale ou politique) devrait tenir compte de l'intérêt des productions dans chacun des milieux, il est plus que vraisemblable que les reproducteurs les moins sensibles aux variations environnementales seront les plus profitables. Plus les milieux de diffusion des reproducteurs seront étendus et variés, meilleure devra être leur robustesse pour les caractères sur lesquels ils sont sélectionnés.

\section{3 / Les hypothèses géné- tiques proposées pour expliquer la canalisation (encadré 1)}

Scheiner et Lyman (1991) rappellent que trois hypothèses polygéniques ont été

Encadré 1. Les trois modèles génétiques proposés pour expliquer les interactions génotype x milieu.

On observe que les performances de l'individu A sont très variables dans 4 environnements différents, alors que celles de l'individu B sont beaucoup plus stables.

\section{Les modèles proposés (Scheiner et Lyman 1991)}

\section{Le modèle de «surdominance»}

Sous cette hypothèse, les individus les plus hétérozygotes sont les mieux armés pour tamponner les effets du milieu.

\section{Le modèle «pléiotropique»}

Les gènes présentent une expression différente dans les différents environnements et la variabilité de ces expressions induit la variabilité des performances. A l'extrême, certains gènes ne s'expriment pas du tout, et des gènes différents s'expriment dans les différents milieux. C'est l'hypothèse explicative la plus classique des interactions $\mathrm{G} \times \mathrm{E}$.

\section{Le modèle «épistatique»}

Des gènes $(\mathrm{R})$ sont sensibles aux variations du milieu et, selon le niveau du milieu, ils modulent l'expression des gènes de production $(\mathrm{P})$.

La variabilité des gènes de sensibilité à l'environnement détermine la variabilité résiduelle des productions.

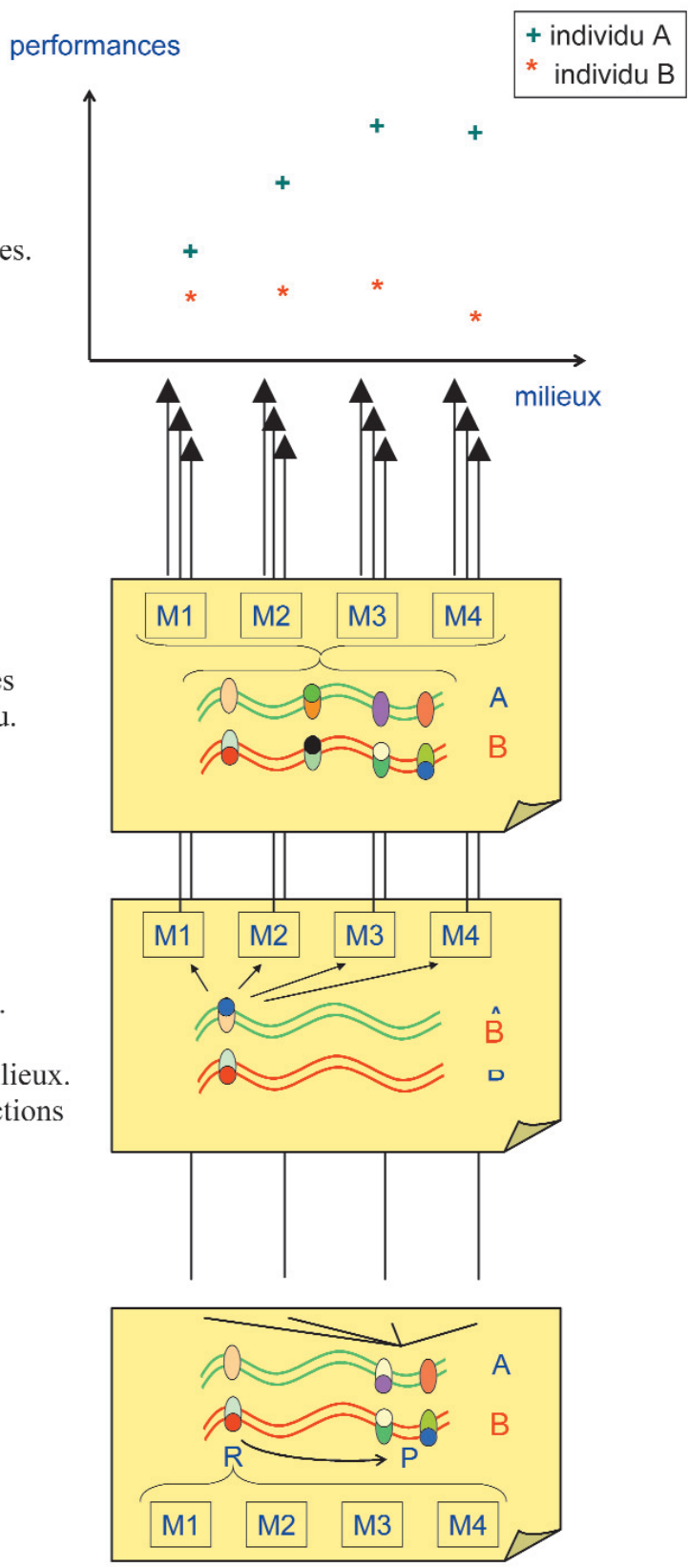


proposées pour expliquer la canalisation d'un caractère et montrent les conséquences qu'auraient respectivement la sélection directionnelle et la sélection canalisante selon chaque hypothèse :

- le modèle de «surdominance» proposé par Lerner (1954), Gillespie et Turelli (1989) suppose que les modifications des phénotypes dans les différents milieux sont fonction du nombre de locus hétérozygotes. L'hétérozygotie offre un plus grand choix de voies métaboliques pour répondre aux sollicitations du milieu et les individus ayant un degré d'homozygotie fort sont moins stables que les hétérozygotes qui possèdent une plus grande capacité d'adaptation. La sélection directionnelle, qui tend à fixer des allèles et augmenter l'homozygotie, conduirait selon cette hypothèse à une diminution de la robustesse.

- le modèle "pléiotropique» repose sur l'hypothèse d'une expression différente des gènes dans différents environnements. Selon cette hypothèse, avancée par Falconer et Mackay (1996), la réponse à une sélection canalisante serait faible mais la sélection directionnelle dans plusieurs milieux induirait une augmentation de la robustesse. A l'extrême, on peut considérer que l'expression d'un gène diminue dans certains milieux au point de ne plus s'exprimer du tout. Ceci permet au modèle pléiotropique d'inclure l'hypothèse génétique classique qui explique les interactions $\mathrm{G} \times \mathrm{E}$ par le fait que des gènes partiellement distincts s'expriment dans les différents milieux.

- selon le modèle «épistatique» (Lynch et Gabriel 1987, Scheiner et Lyman 1989), les gènes qui déterminent l'expression d'un caractère sont partiellement différents de ceux qui contrôlent sa variabilité environnementale. La réponse à la sélection pour la canalisation dépendra de l'héritabilité de ce caractère et la réponse corrélée à la sélection directionnelle dépendra de la corrélation entre les deux groupes de gènes qui n'est pas connue a priori.

Ces trois hypothèses génétiques ne sont évidemment pas exclusives et peuvent coexister. Ils ne sont pas non plus les seules possibles, de sorte que comme le remarquent Scheiner et Lyman (1991), «il n'y a pas de raison pour que la nature dans sa perversité ne nous fournisse pas des résultats contradictoires»».

\section{4 / Les modèles mathéma- tiques (encadré 2)}

D'une manière générale, pour tester des hypothèses, rechercher des facteurs de variation ou estimer des valeurs génétiques, les généticiens construisent des modèles mathématiques qui reposent sur des hypothèses génétiques spécifiques au problème posé. Pour analyser les interactions génotype $\times$ milieu, ils disposent en particulier de plusieurs types de modèles mathématiques adaptés aux modèles génétiques présentés précédemment. Par exemple, un modèle multicaractère correspond bien à l'hypothèse pléiotropique, alors que les modèles de norme de réaction sont particulièrement appropriés lorsqu'on suspecte des gènes de sensibilité à l'environnement comme dans le modèle épistatique. Les modèles structuraux, quant à eux, répondent bien à l'hypothèse de gènes qui contrôlent la variabilité des caractères. Toutefois, ce principe général est loin d'être figé et le choix d'un modèle peut répondre à d'autres contraintes. En pratique, il est notamment largement conditionné par la nature et le nombre de milieux entrant dans l'interaction.

\section{1 / Cas d'environnements peu nombreux}

Dans le cas où le nombre de milieux concernés par les interactions est faible $(<5)$, on peut estimer les valeurs génétiques des individus pour chaque milieu sans difficulté particulière, par exemple en utilisant un modèle multicaractère. La sélection peut être faite en utilisant les valeurs génétiques spécifiques à chaque milieu dans un index global dans lequel les caractères sont pondérés en fonction de leur valeur économique. Néanmoins, on peut ignorer l'existence de ces interactions mais veiller à ce que les animaux qui servent à l'évaluation s'expriment également dans tous les environnements. Ainsi, pour les ovins laitiers de Sardaigne, une analyse multicaractère correspondant à la production de lait dans trois grands types de combinaison troupeaux $\times$ années (faible, moyenne et forte production) a mis en évidence des valeurs génétiques des pères différentes selon les milieux (Sanna et al 2002). Cela justifierait de considérer trois caractères, mais scinder le schéma de sélection en trois ne semble pas une solution souhaitable. La meilleure option pour fournir aux éleveurs des génotypes adaptés à une bonne expression des phénotypes dans les différentes conditions est de sélectionner les reproducteurs dans les trois milieux.

Dans le cas où le nombre de milieux devient important, le nombre de valeurs génétiques estimées avec le modèle précédent est trop important pour chaque individu et leur utilisation pour l'amélioration génétique est difficile. Du point de vue mathématique, l'enjeu est alors de synthétiser au mieux l'ensemble des interactions génotype $\times$ milieu, notamment en réduisant au maximum le nombre de paramètres à estimer.

\section{2 / Cas de milieux identifiés}

Si les milieux sont identifiés, on peut utiliser les normes de réaction qui résument les variations de chaque génotype dans les différents milieux par une fonction de quelques paramètres (fonction polynomiale par exemple). Les valeurs génétiques associées aux paramètres de cette fonction permettent de caractériser la valeur moyenne de l'individu et sa sensibilité à l'environnement. Bien que ces normes de réaction aient été conçues pour des variations de milieux continues (gradient de température, quantité de protéines dans la ration...), cette méthodologie peut être étendue au cas d'effets non continus (facteur «élevage» par exemple) en introduisant dans le modèle la valeur de chaque niveau de l'effet estimée préalablement ou en se basant sur les propositions méthodologiques récentes de $\mathrm{Su}$ et al (2006) qui estiment et utilisent cette valeur dans le même modèle.

\section{3 / Cas de milieux non identi- fiés}

Si les milieux ne sont pas identifiés ou sont incontrôlables comme les micro-variations environnementales, on utilise des modèles structuraux de la variance qui décomposent dans un même modèle le niveau des performances et leur variabilité. Dans ces modèles (développés en détail ci-dessous) les interactions entre génotype et milieu sont résumées sous la forme d'une seule valeur génétique traduisant la variabilité des performances d'un génotype dans différents milieux.

La mise en oeuvre des modèles mathématiques structuraux est complexe. Une méthode simplifiée permet une estimation très rapide mais approximative des paramètres. Cette dernière revient à estimer dans un premier temps les paramètres qui agissent sur la moyenne du caractère et à calculer la variance des résidus des observations de chaque individu puis à estimer, dans un deuxième temps, les effets qui agissent sur cette variabilité. Cette méthode a été utilisée dans un grand nombre d'expériences tant en France qu'à l'étranger. Certains auteurs se limitent même à la deuxième étape en analysant directement l'écart-type des observations. Ce qui revient à ignorer dans le calcul de cet écart-type tous les facteurs environnementaux et génétiques qui affectent la moyenne. Plus récemment, un logiciel permettant une estimation simultanée de l'ensemble des paramètres du modèle a été développé et mis à disposition 
Encadré 2. Les différents modèles mathématiques de prise en compte des interactions $G \times E$.

On peut admettre qu'il y a interaction génotype $\mathrm{x}$ milieu lorsque des effets non génétiques modifient les performances de façon différente selon les génotypes. Cela regroupe trois hypothèses génétiques différentes auxquelles sont associées des modèles mathématiques qui permettent de les traiter.

Le modèle le plus simple consiste à estimer une valeur génétique pour chaque environnement, par exemple à l'aide d'un modèle multicaractère.

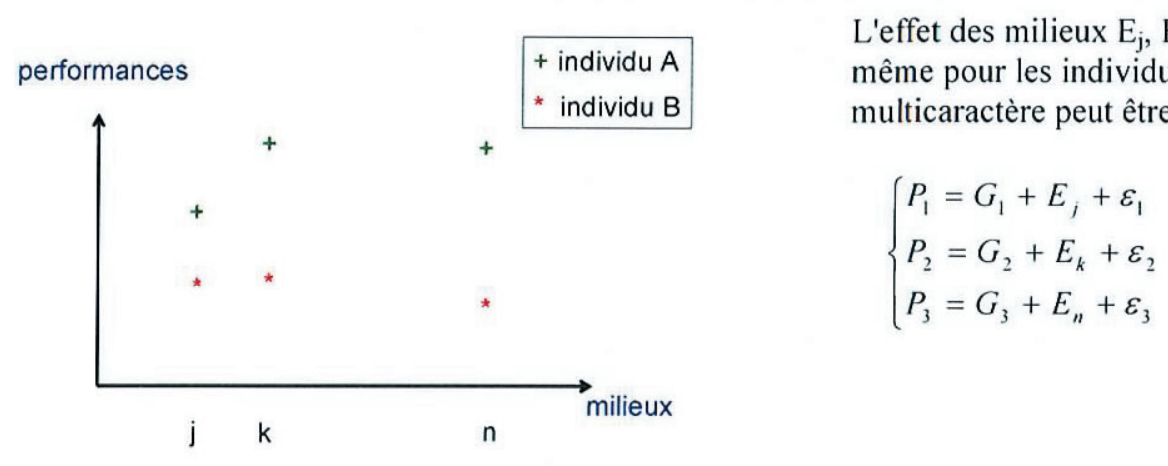

Lorsque les génotypes sont placés dans un grand nombre de milieux, on ne peut plus estimer et utiliser une valeur génétique pour chaque milieu. Il existe alors deux façons de réduire le nombre de paramètres :

a) les normes de réaction, qui ajustent les performances de chaque individu dans les différents milieux par une fonction de quelques paramètres (une fonction polynomiale par exemple) et procurent les valeurs génétiques pour la moyenne et pour la sensibilité à l'environnement.

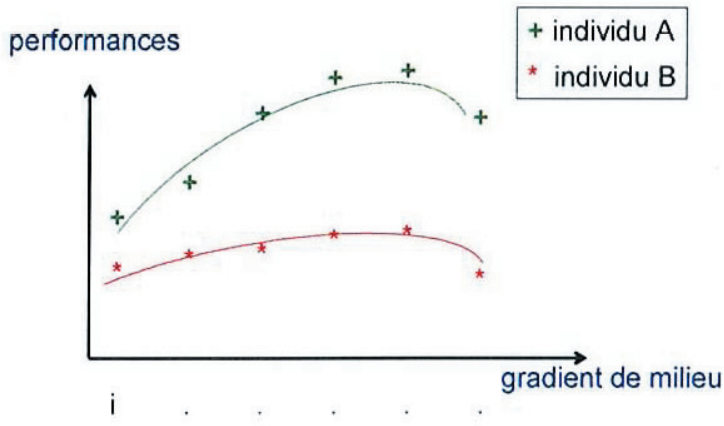

La sensibilité aux variations de l'environnement est plus faible pour B que pour A. Un modèle de régression est bien adapté.

$$
P=G_{0}+G_{1} \times E+G_{2} \times E^{2}+\ldots .+\varepsilon
$$

b) les modèles structuraux, qui décomposent non seulement la moyenne mais aussi la variance des performances en effets génétiques, environnementaux et résiduels. Ils sont particulièrement adaptés aux facteurs environnementaux non identifiés (résiduels) mais l'hypothèse génétique sous-jacente permet de les utiliser même pour des effets environnementaux identifiés ayant un très grand nombre de niveaux.

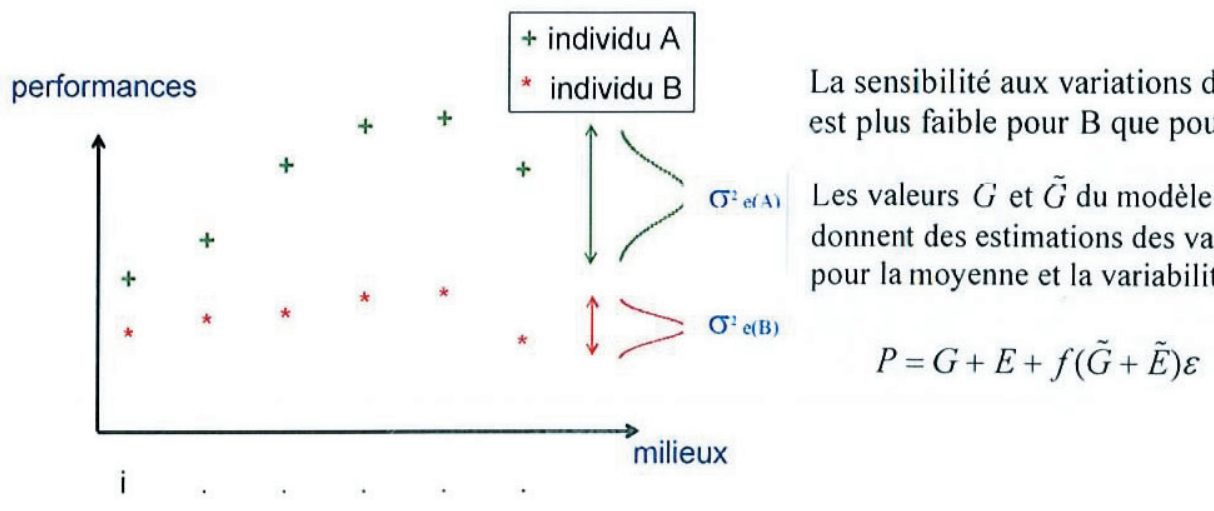


Figure 1. Représentation des valeurs génétiques en unité d'écart-type, estimées sur descendance pour la moyenne (u) et la variabilité (v) de la prolificité de 157 mâles Lacaune (San Cristobal et al 2001).

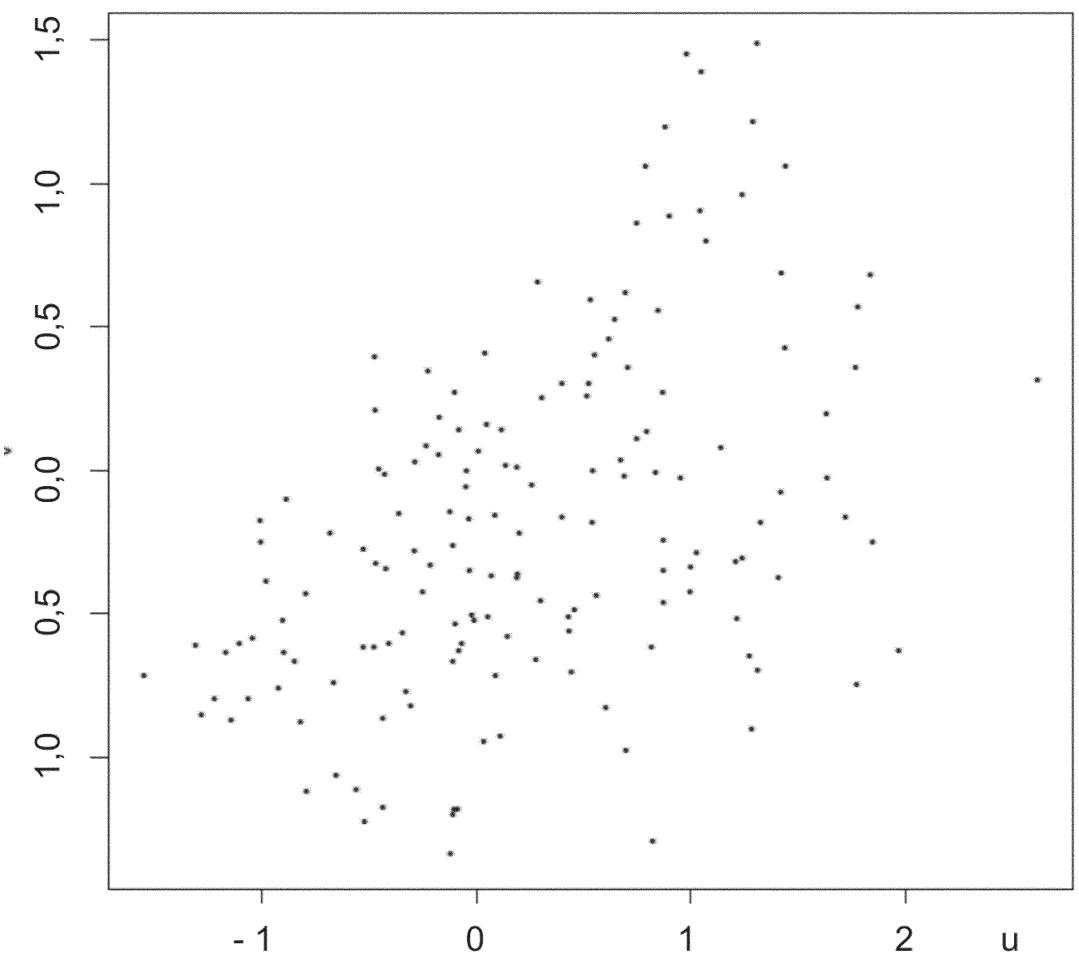

du public (Garcia et Ibañez-Escriche 2009). Néanmoins, la méthode de Monte-Carlo par chaîne de Markov qui est utilisée dans ce logiciel présente l'inconvénient de nécessiter des temps de calcul très longs (de l'ordre de deux jours pour un millier d'animaux mesurés chacun une vingtaine de fois). La mobilisation informatique qu'impose cette méthode proscrit son utilisation pour l'estimation en routine des valeurs génétiques dans des populations importantes. De plus, un tel modèle nécessite une grande quantité d'informations pour obtenir une précision suffisante des estimées de valeurs génétiques. Par exemple, des familles paternelles de 50 à 100 descendants peuvent être nécessaires pour assurer une estimation suffisamment précise dans le cas d'une seule mesure phénotypique par individu (Mulder et al 2007). La figure 1 montre, par exemple, la répartition conjointe des valeurs génétiques de 157 béliers Lacaune pour la moyenne et la variabilité de la prolificité de leurs filles.

\section{5 / Peut-on réellement sélec- tionner des animaux moins variables?}

\section{1 / Utilisation du croisement ou sélection en race pure}

De très nombreux exemples (cf. Falconer et Mackay 1996) montrent la plus faible sensibilité aux variations environnementales des animaux croisés par rapport aux lignées consanguines, que ce soit chez les plantes ou les animaux de laboratoire où de telles lignées existent. Cependant, ce n'est pas un phénomène universel et, pour les animaux de rente, il n'est pas démontré que l'augmentation d'hétérozygotie amenée par le croisement entre des races ou lignées très peu consanguines conduit systématiquement à une plus grande stabilité des productions. Par ailleurs, à moins de sélectionner les races pures pour l'aptitude au croisement en termes de variabilité, il n'est pas possible d'envisager une sélection. S'il est peu vraisemblable que le croisement soit choisi comme méthode pour améliorer génétiquement la robustesse d'un caractère particulier, on peut imaginer que dans quelques situations spécifiques de gestion génétique de l'aptitude au croisement (sélection des races parentales et production des animaux croisés sous la dépendance du même sélectionneur) un contrôle de la robustesse des productions soit mis en œuvre.

Il faut aussi noter que l'uniformité génétique bien connue des lignées consanguines ne signifie pas qu'ils sont moins sensibles aux variations environnementales. Il est particulièrement important de distinguer la variabilité de l'expression d'un caractère par différents individus d'une même population dans un environnement unique, de la variabi- lité des performances des individus de cette même population dans différents environnements.

Une littérature abondante rapporte les résultats d'expériences de sélection visant à réduire la variabilité des caractères. Mais elles concernent principalement des espèces de laboratoire comme la drosophile (Rendel et Sheldon 1960, Waddington 1960, Prout 1962, Piniero 1992), le Tribolium (Kauffman et al 1977, Cardin et Minvielle 1986) ou la souris (Rendel 1979). La plupart du temps, la sélection était stabilisatrice c'est-à-dire qu'elle tendait à éliminer les formes extrêmes d'un caractère au bénéfice des individus moyens. Des expériences de sélection canalisante ont été mises en place par Waddington (1960) et Scheiner et Lyman (1991) dans le but de sélectionner des individus moins sensibles à la température du milieu d'élevage ainsi que par Gibson et Bradley (1974) qui ont réalisé une élimination des individus extrêmes dans une population élevée dans un environnement instable. Les trois principales conclusions de ces expériences sont : 1) la sélection est généralement efficace et conduit à une diminution importante de la variance phénotypique ; 2) les valeurs d'héritabilité estimées au début et à la fin des expériences montrent assez souvent que la variance génétique du caractère sélectionné diminue légèrement ; 3) dans beaucoup de cas, la variance environnementale ou la sensibilité des individus aux fluctuations de l'environnement est effectivement réduite. Cela montre finalement que la sélection pour la robustesse de caractères zootechniques (définie comme la sensibilité de leur expression vis-à-vis des effets du milieu) doit être possible et efficace.

\section{2 / Variabilité phénotypique et génétique de la robustesse des caractères ; valeurs génétiques et sélection}

L'existence d'une variabilité phénotypique de la sensibilité aux effets du milieu est bien connue. Par exemple, Rowe et al (2006) montrent qu'il subsiste une hétérogénéité des variances résiduelles des poids de poulet à 35 jours entre des groupes d'accouplement, après correction pour les facteurs de variation qui agissent sur la moyenne du caractère (figure 2). Une analyse appropriée montre que cette hétérogénéité provient d'une différence génétique. D'autres preuves d'une variabilité génétique de la variance environnementale dans les espèces domestiques ont été rapportées à propos de la taille de portée des brebis (San Cristobal-Gaudy et al 2001, Amer et Bodin 2006) ou des truies (Sorensen et Waagepetersen 2003). 
Figure 2. Hétérogénéité des variances résiduelles $\left(Z_{g}\right)$ du poids de 47730 poulets mâles de 35 jours issus de 50 groupes d'accouplement. La ligne pointillée indique la valeur attendue de $Z_{g}$ en l'absence d'hétérogénéité, les cercles montrent les valeurs de $Z_{g}$ après exclusion des données aberrantes (> 2,7 s). D'après Rowe et al (2006).

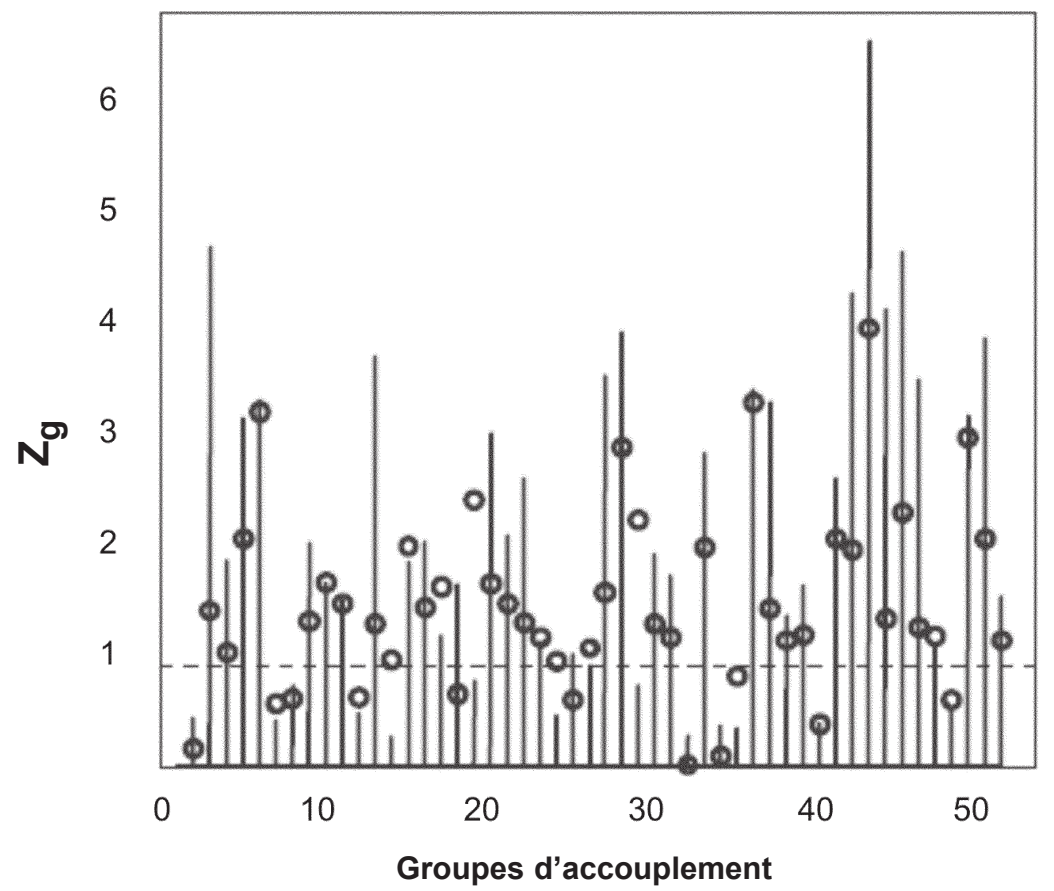

Les paramètres génétiques de la robustesse de plusieurs caractères ont été estimés. Quelles que soient les espèces et les méthodes d'estimation, la variance génétique additive de la robustesse des caractères est toujours beaucoup plus faible que celle de la moyenne des mêmes caractères, mais elle est aussi généralement significativement différente de zéro. Les héritabilités estimées sont aussi toujours très faibles (tableau 1) mais leur signification ne peut être comparée à celle de la moyenne des caractères ; elles n'ont notamment pas la propriété d'être le coefficient de régression de la valeur génétique additive sur la valeur phénotypique.

L'ensemble de ces études montre qu'il existe une part génétique non négligeable dans l'hétérogénéité de la variance résiduelle de nombreux caractères. Cette hétérogénéité peut être interprétée comme une variabilité génétique de la sensibilité environnementale de telle sorte que les génotypes les plus sensibles aux microvariations de l'environnement (modifications marginales des températures, de l'alimentation, voire des sources de stress) présentent des variances résiduelles plus grandes que les génotypes moins sensibles aux mêmes variations du milieu. D'une manière générale, la plasticité et la canalisation naturelle de certains caractères peuvent être expliquées par un contrôle génétique de la sensibilité aux variations environne- et variabilité) dans l'index global et les corrélations génétiques avec les autres caractères d'intérêt (sélectionnés ou non) sont aussi déterminantes. Si l'estimation des corrélations génétiques entre caractères est toujours délicate, elle est encore plus complexe entre la moyenne d'un caractère et sa variabilité. En effet, au-delà de la liaison biologique entre les deux variables, il existe une relation mathématique qui lie la moyenne d'un caractère à sa variance, ce qui ajoute une complexité supplémentaire. Parmi les quelques estimations de corrélations publiées, certaines sont proches de 1 (Gutierrez et al 2006) et sont en partie expliquées par l'existence de cette relation mathématique ou facteur d'échelle. Récemment, Yang et al (2009) ont proposé une méthode de recherche et de correction du facteur d'échelle dans une analyse de données pour la canalisation en utilisant une transformation BoxCox. L'utilisation de cette transformation diminue fortement la corrélation mais augmente son incertitude de façon très importante. D'autres estimations sont au contraire voisines de zéro (Garreau et al 2008), voire même proche de - 1 (Gutierrez et al 2006). Ces résultats peu cohérents montrent les difficultés d'estimation des paramètres de tels modèles.

mentales lorsque la fitness dépend de la capacité à s'adapter ou du contrôle de l'expression phénotypique ; en effet, la variabilité ou la stabilité de l'expression du caractère est alors directement sélectionnée.

Toutefois, l'intérêt d'inclure la canalisation d'un caractère dans l'objectif de sélection ne dépend pas uniquement de l'héritabilité de sa variabilité. Les pondérations de chaque caractère (moyenne

Par ailleurs, l'espérance du progrès génétique réalisable dans le cadre d'une sélection visant à réduire la sensibilité d'un caractère aux micro-variations environnementales et le canaliser autour d'une valeur optimum est difficile à calculer de façon analytique. Une démarche dynamique assurant à chaque génération les meilleures pondérations de la moyenne et de la variabilité dans l'index

Tableau 1. Héritabilité de la moyenne et de la variabilité des caractères dans diverses expérimentations.

\begin{tabular}{|l|c|c|c|}
\hline Caractères & $\begin{array}{c}\text { Héritabilité } \\
\text { de la } \\
\text { moyenne }\end{array}$ & $\begin{array}{c}\text { Héritabilité } \\
\text { de la } \\
\text { variabilité }\end{array}$ & Auteurs \\
\hline Poids de naissance des porcelets & - & 0,08 & $\begin{array}{c}\text { Damgaard et al } \\
(2003)\end{array}$ \\
\hline Poids de naissance des porcelets & 0,32 & 0,10 & $\begin{array}{c}\text { Huby et al } \\
(2003)\end{array}$ \\
\hline Poids des poulets vers 35 jours & 0,35 & 0,05 & $\begin{array}{c}\text { Mulder et al } \\
(2009)\end{array}$ \\
\hline Poids des poulets vers 45 jours & 0,21 & 0,03 & $\begin{array}{c}\text { Wolc et al } \\
(2009)\end{array}$ \\
\hline Poids de naissance des lapereaux & 0,06 & 0,01 & $\begin{array}{c}\text { Garreau et al } \\
(2008)\end{array}$ \\
\hline Poids de naissance des porcelets & 0,06 & 0,05 & $\begin{array}{c}\text { Canario et al } \\
(2010)\end{array}$ \\
\hline
\end{tabular}


de sélection doit être envisagée pour optimiser la réponse globale, compte tenu du nombre de générations prévues, de la quantité d'information et de tous les paramètres génétiques et économiques. Quelques auteurs ont proposé des solutions et ont montré, par exemple, qu'il serait possible d'obtenir en une génération de sélection une réduction de 20 à $30 \%$ de la variance environnementale du poids des poulets femelles aux alentours de 45 jours en sélectionnant uniquement sur ce caractère (Mulder et al 2009). Toutefois, les incertitudes restantes et les problèmes que pose l'estimation de cette réponse prédite confèrent beaucoup d'intérêt aux résultats des diverses expériences de sélection.

\section{3 / Exemples de sélection cana- lisante chez les animaux de ferme}

Nous avons vu plus haut que de nombreuses expériences de sélection canalisante ont été entreprises chez les espèces de laboratoire, alors que pour les animaux de rente, il n'y a à notre connaissance que trois expériences qui ont été publiées à ce jour. Elles ont toutes été entreprises en France et ont concerné respectivement l'homogénéité du poids adulte de l'escargot, la variabilité intra-portée du poids des lapereaux à la naissance et la variabilité du $\mathrm{pH}$ ultime de la viande de porc. Bien qu'ayant produit des résultats mitigés, elles permettent de tirer un certain nombre de conclusions. Les résultats de deux de ces expériences sont rapportés ici.

a) Canalisation du poids à la naissance chez le lapin

Une expérience de sélection divergente sur la variabilité du poids de naissance des lapereaux intra-portée a été mise en place en 2002 afin d'étudier les possibilités d'une sélection canalisante et d'en mesurer les réponses directes et indirectes, notamment sur la mortinatalité (Garreau et al 2008). Les deux lignées d'environ cent femelles chacune ont été constituées à partir d'une souche commerciale indexée sur la variabilité intra-portée du poids à la naissance des lapereaux. Le caractère est attribué aux femelles ayant de 3 à 5 portées qui permettent donc d'obtenir autant de performances de variabilité des poids individuels à la naissance. L'évaluation génétique, effectuée à chaque génération selon un modèle structural simplifié, fournit des valeurs individuelles pour le poids moyen de la portée et pour sa variabilité. La figure 3 illustre l'évolution régulière de l'écart-type des poids de naissance au cours des générations. Après 10 générations de sélection, l'écart-type de ce poids est de 7,34 g
Figure 3. Evolution au cours des générations de l'écart-type du poids à la naissance des lapereaux dans des lignées sélectionnées de façon divergente sur la variabilité intra-portée de ce caractère. En noir, figurent les droites de régression de l'écart-type sur le numéro de génération (Bodin et al 2010).

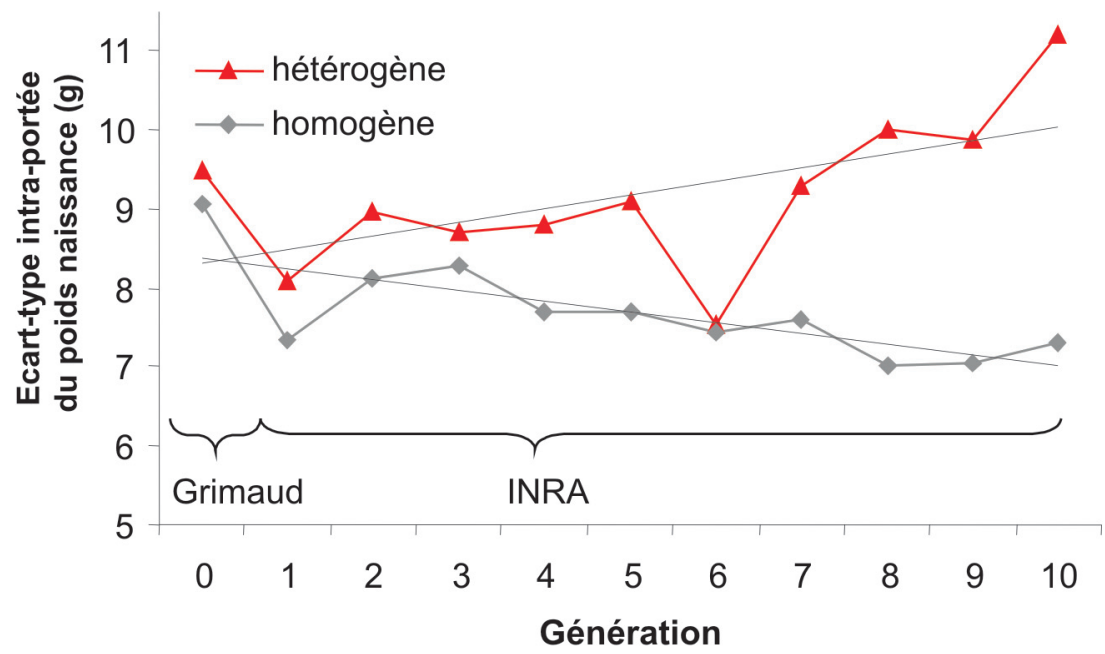

dans la lignée homogène contre 11,26 $\mathrm{g}$ dans la lignée hétérogène $(\mathrm{P}=0,01)$. Au cours de cette période, l'écart-type phénotypique de la lignée homogène a diminué de $16 \%$. Une estimation récente des paramètres génétiques dans cette population montre que les variances génétiques additives pour le poids des lapereaux à la naissance et sa variabilité sont significativement différentes de zéro. La corrélation entre ces deux variables est très proche de zéro, ce qui explique l'absence d'évolution consistante et significative des poids à la naissance. Dès la quatrième génération de sélection, la mortalité des lapereaux entre la naissance et le sevrage était significativement plus faible dans la lignée homogène $(17,7$ contre $32,7 \%)$. On a aussi observé que les cornes utéri-

nes étaient significativement plus longues et présentaient une capacité d'élongation plus importante dans la lignée homogène (Bolet et al 2007). Dès la cinquième génération, cette expérience a montré que la sélection canalisante d'un caractère était possible et pouvait être efficace. Elle est maintenant pratiquée en routine par le détenteur de la souche concernée.

b) Canalisation du pH ultime de la viande de porc

Le $\mathrm{pH}$ ultime du muscle mesuré $24 \mathrm{~h}$ après l'abattage est un facteur important de la qualité technologique de la viande de porc, utilisé comme critère de tri des jambons avant transformation. Des analyses préalables avaient montré que la

Figure 4. Evolution de l'écart-type du pH ultime du muscle demi-membraneux des carcasses de porcs Large White des lignées hétérogène $(H)$ et homogène $(B)$ au cours de 5 générations de sélection divergente (d'après Larzul et al 2006).

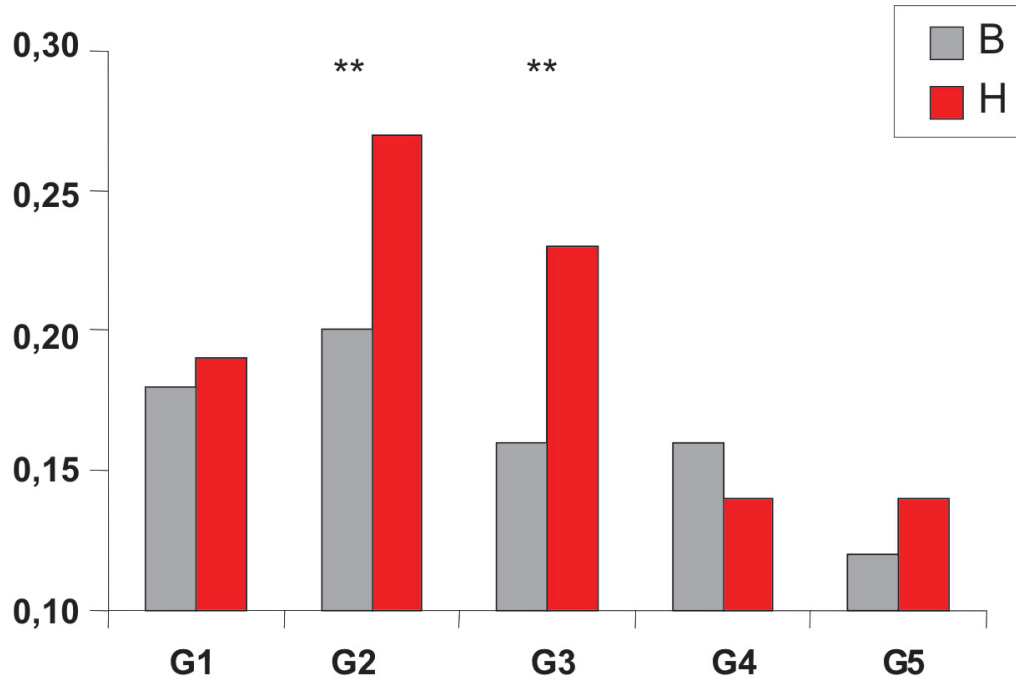


variabilité intra-famille de ce phénotype variait de 1 à 10 selon les familles paternelles en race Large White (San Cristobal-Gaudy et al 1998b). A des fins de démonstration, une sélection divergente en lignées ouvertes a donc été entreprise dans cette race pour laquelle un contrôle en routine du $\mathrm{pH}$ ultime des carcasses de porcs permet d'estimer chaque année les valeurs génétiques d'environ 300 verrats pour le $\mathrm{pH}$ ultime et sa variabilité. A chaque génération, six mâles ayant une valeur de la moyenne proche de l'optimum $(\mathrm{pH}=5,7)$ mais des valeurs extrêmes pour la variabilité du $\mathrm{pH}$ ultime du muscle demi-membraneux (3 «homogènes» et 3 «hétérogènes») servaient à inséminer chacun 6 femelles des lignées haute et basse de l'unité expérimentale INRA de Bourges. Les porcelets issus de ces femelles étaient engraissés, abattus et le $\mathrm{pH}$ ultime de leur muscle semi-membraneux mesuré. Dans chaque lignée, une fille était retenue au hasard pour remplacer sa mère. Ce type de schéma ouvert permet d'accumuler rapidement les gènes favorables détectés dans une lignée. Conformément à l'objectif poursuivi, le $\mathrm{pH}$ ultime moyen n'a pas évolué au cours des générations de sélection. En revanche, l'écart-type de ce caractère était plus faible dans la lignée homogène que dans la lignée hétérogène pour les générations 2 et 3 (figure 4 , Larzul et al 2006). Ce résultat n'a malheureusement pas été confirmé dans les générations suivantes où l'incohérence de certains paramètres a fait suspecter un facteur inconnu non contrôlé.

\section{Conclusion}

Dans cet article, les notions de robustesse et de canalisation ont été présentées sous l'angle génétique. La différence de sensibilité des individus aux variations de milieu qui induit une variabilité de leurs performances a été décrite comme résultant de l'interaction entre le génotype et le milieu. L'axe corticotrope a un rôle majeur dans la réponse non spécifique d'un animal face à des situations de stress mais la robustesse doit aussi être considérée comme une propriété d'un caractère de production donné et, à ce titre, nous conduit à nous intéresser à la distribution de ce caractère.

Plusieurs modèles génétiques peuvent expliquer la robustesse des caractères. Nous en avons sommairement explicité quelques-uns, mais il en existe d'autres. Tous les modèles possibles ne sont évidemment pas exclusifs et peuvent coexister pour assurer la stabilité d'un caractère. Les modélisations mathématiques qui sont faites répondent d'une part à des hypothèses génétiques et biologiques mais aussi aux contraintes pratiques liées à l'utilisation des valeurs génétiques. Beaucoup de problèmes restent à résoudre. Par exemple, dans le cas des modèles structuraux, un écart à la normalité de la distribution des données conduit à des biais dans les estimations de la corrélation entre les valeurs génétiques influençant la moyenne ou la variance, et ces biais sont difficiles à corriger. Par ailleurs, il est également difficile d'appréhender le comportement d'un tel modèle face à l'existence de QTL (plutôt de gènes majeurs) qui agissent sur la moyenne du caractère concerné et en l'absence de répétition des observations par individu. Les apports de la génétique moléculaire seront, à ce titre, importants puisqu'ils permettront de lever la confusion entre la variabilité des performances observée entre les individus d'une famille ou d'une population due à la ségrégation de QTL qui contrôlent la moyenne ou à la ségrégation de gènes qui affectent directement la variabilité.

La prise en compte de la robustesse d'un caractère dans les objectifs de sélection nécessite de pondérer à chaque génération les efforts à faire sur la moyenne et sur la variabilité du caractère. Là encore, de nombreuses interrogations restent en suspens sur la stratégie de sélection optimale et sur les conséquences possibles de cette dernière en termes de variabilité génétique. C'est pourquoi la recherche génétique dans ce domaine est en plein essor, motivée par des expériences réussies de canalisation.

\section{Remerciements}

Les auteurs remercient les lecteurs pour leurs remarques et commentaires pertinents.

\section{Références}

Amer P.R., Bodin L., 2006. An evaluation of the scope for quantitative genetic selection for twinning rate in ewes. Proc. N. Z. Soc. Anim. Prod., 22-26 June, Napier, Nouvelle-Zélande, $66,429-433$

Bodin L., Garcia M., Bolet G., Garreau H. 2010. Results of canalizing selection for rabbit birth weight. Proc. $9^{\text {th }}$ WCGALP, Liepzig, Germany, 2-6 august 2010, à paraître.

Bolet G., Garreau H., Joly T., Theau-Clement M., Falières J., Hurtaud J., Bodin L., 2007. Genetic homogenisation of birth weight in rabbits: indirect selection response for uterine horn characteristics. Livest. Sci., 111, 28-32.

Brown K.I., Nestor K.E., 1973. Some physiological responses of turkeys selected for high and low adrenal response to cold stress. Poult. Sci., 52, 1948-1954.

Canario L., Lundgren H., Haandlykken M., Rydhmer L., 2010. Genetics of growth in piglets and the association with homogeneity of body weight within litters. J. Anim. Sci., 88, 1240-1247.

Cardin S., Minvielle F., 1986. Selection on phenotypic variation of pupa weight in Tribolium castaneum. Can. J. Genet. Cytol., $28,856-861$
Damgaard L.H., Rydhmer L., Lovendahl P. Grandinson K., 2003. Genetic parameters for within-litter variation in piglet birth weight and change in within-litter variation during suckling. J. Anim. Sci., 81, 604-610.

Debat V., David P., 2001. Mapping phenotypes: canalization, plasticity and developmental stability. Trends Ecol. Evol., 16, 555-561.

Falconer D.S., Mackay T.F.C., 1996. Introduction to quantitative genetics. Longmans Green, Harlow, Essex, UK, $4^{\text {th }}$ Edition, 464p.

Foury A., Geverink N.A., Gil M., Gispert M., Hortos M., Furnols M.F.I., Carrion D. Blott S.C., Plastow G.S., Mormede R., 2007. Stress neuroendocrine profiles in five pig breeding lines and the relationship with carcass composition. Animal, 1, 973-982.

Freeman G.H., 1973. Statistical methods for the analysis of genotype-environment interactions. Heredity, 31, 339-354.

Garcia M., Ibañez-Escriche N., 2009 GSEVM v.3: MCMC software to analyze genetically structured environmental variance models. Proc. $60^{\text {th }}$ Ann. Meet. EAAP, 24-27 August, Barcelona, Spain, 1p.

Garreau H., Bolet G., Larzul C., RobertGranié C., Saleil G., SanCristobal M., Bodin
L., 2008. Results of four generations of a canalising selection for rabbit birth weight. Livest. Sci., 119, 55-62.

Gibson J.B., Bradley B.P., 1974. Stabilising selection in constant and fluctuating environments. Heredity, 33, 293-302.

Gillespie J.H., Turelli M., 1989. Genotypeenvironment interaction and the maintenance of polygenic variation. Genetics, 121, 129-138.

Gross W.B., Siegel P.B., 1985. Selective breeding of chickens for corticosterone response to social stress. Poult. Sci., 64, 2230-2233.

Gutierrez J.P., Nieto B., Piqueras P., Ibañez N., Salgado C., 2006. Genetic parameters for canalisation analysis of litter size and litter weight traits at birth in mice. Genet. Sel. Evol., $38,445-462$.

Huby M., Gogué J., Maignel L., Bidanel J.P., 2003. Corrélations génétiques entre les caractéristiques numériques et pondérales de la portée, la variabilité du poids des porcelets et leur survie entre la naissance et le sevrage. Journ. Rech. Porcine Fr., 35, 293-300.

Kauffman P.K., Enfield F.D., Comstock R.E., 1977. Stabilizing selection for pupa weight in Tribolium castaneum. Genetics, 87, 327-341. 
Knap P.W., 2005. Breeding robust pigs. Aust. J. Exp. Agric., 45, 763-773.

Larzul C., Le Roy P., Tribout T., Gogue J., SanCristobal M., 2006. Canalyzing selection on ultimate $\mathrm{PH}$ in pigs: consequences on meat quality. Proc. $8^{\text {th }}$ WCGALP, August 13-18, Belo Horizonte, Brasil, $4 p$.

Leenhouwers J.I., Knol E.F., de Groot P.N., Vos H., van der Lende T., 2002. Fetal development in the pig in relation to genetic merit for piglet survival. J. Anim. Sci., 80, 1759-1770.

Lerner I.M., 1954. Genetic homeostasis. Oliver and Boyd, London, UK, 134p.

Lynch M. Gabriel W., 1987. Environmental tolerance. Am. Nat., 129, 283-303.

Michel V., Peinnequin A., Alonso A., Buguet A., Cespuglio R., Canini F., 2007. Decreased heat tolerance is associated with hypothalamopituitary-adrenocortical axis impairment. Neurosci., 147, 522-531

Minozzi G., Guémené D., Couty M., Gourichon D., Minvielle F., Pinard-van der Laan M.H., 2008. Circulating corticosterone reaction to restraint and adrenocorticotropin hormone administration in white leghorns selected for immune response traits. Poult Sci., 87, 2225-2230

Mulder H.A., Bijma P., Hill W.G., 2007. Prediction of breeding values and selection responses with genetic heterogeneity of environmental variance. Genetics, 175, 1895-1910.

Mulder H.A., Hill W.G., Vereijken A., Veerkamp R.F., 2009. Estimation of genetic variation in residual variance in female and male broiler chickens. Animal, 3, 1673-1680.
Piniero R., 1992. Selection for canalization at two dorsocentral bristles in Drosophila melanogaster. J. Heredity, 83, 49-55.

Pottinger T.G. Carrick T.R., 2000. Indicators of reproductive performance in rainbow trou Oncorhynchus mykiss (Walbaum) selected for high and low responsiveness to stress. Aquac. Res., 31, 367-375.

Prout T., 1962. The effects of stabilizing selection on the time of development in Drosophila melanogaster. Genet. Res., 3, 364-382.

Rendel J.M., 1979. Canalisation and selection. In: Quantitative genetic variation. Thompson J.N., Thoday J.M. (Eds), Academic Press, New York, 139-156.

Rendel J.M., Sheldon B.L., 1960. Selection for canalization of the scute phenotype in Drosophila melanogaster. Austr. J. Biol. Sci., 13, 36-47.

Rowe S.J., White I.M.S., Avendano S., Hill W.G., 2006. Genetic heterogeneity of residua variance in broiler chickens. Genet. Sel. Evol. $38,617-635$.

San Cristobal-Gaudy M., Elsen J.M., Bodin L., Chevalet C., 1998a. Prediction of the response to a selection for canalisation of a continuous trait in animal breeding. Genet. Sel. Evol., 30, 423-451.

San Cristobal-Gaudy M., Elsen J.M., Bodin L., Chevalet C., Manfredi E., Le Roy P., 1998b. Prediction of the response to canalising selection of a continuous trait. Proc. $6^{\text {th }}$ WCGALP, Armidale, Australia, 26, 85-88.

San Cristobal-Gaudy M., Bodin L., Elsen J.M., Chevalet C., 2001. Genetic components of litter size variability in sheep. Genet. Sel. Evol., 33, 249-271.
Sanna S.R., Carta A., Casu S., 2002 Genotype by environment interaction for milk yield in Sarda dairy sheep. J. Anim. Breed. Genet., 119, 190-199.

Scheiner S.M., Lyman R.F., 1989. The Genetics of phenotypic plasticity. 1. Heritability. J. Evol. Biol., 2, 95-107.

Scheiner S.M., Lyman R.F., 1991. The genetics of phenotypic plasticity. 2. Response to selection. J. Evol. Biol., 4, 23-50.

Sorensen D., Waagepetersen R., 2003. Normal linear models with genetically structured residual variance heterogeneity: a case study. Genet. Res., 82, 207-222.

Su G., Madsen P., Lund M.S., Sorensen D., Korsgaard I.R., Jensen J., 2006. Bayesian analysis of the linear reaction norm model with unknown covariates. J. Anim. Sci., 84, 16511657

Waddington C.H., 1940. The genetic control of wing development in Drosophila . J. Genet., 41, 75-139.

Waddington C.H., 1960. Experiments on canalizing selection. Genet. Res., 1, 140-150.

Wolc A., White I.M.S., Avendano S., Hill W.G., 2009. Genetic variability in residual variation of body weight and conformation scores in broiler chickens. Poult. Sci., 88, 1156-1161.

Yang Y., Christensen O.F., Sorensen D. 2009. Analysis of genetically structured variance heterogeneity and the Box-Cox transformation. $60^{\text {th }}$ Annual Meeting of the EAAP, 2427. August, Barcelona, Spain, 1p.

\section{Résumé}

L'objectif de cet article est de définir et illustrer la notion de robustesse telle qu'elle est vue par les généticiens. Pour ces derniers, il existe deux concepts de robustesse. Le premier considère que la robustesse correspond à la capacité de survie de l'animal et au maintien d'un potentiel global de production dans des environnements stressants et fait notamment référence au rôle de l'axe corticotrope. Le second concept définit la robustesse comme le maintien d'un potentiel de production spécifique : dans ce cadre, un animal est dit robuste pour un caractère donné si ce caractère est stable dans des environnements variés, ce qui rejoint la notion d'interaction génotype-milieu et s'explique par différentes hypothèses génétiques (modèle de surdominance, modèle pléiotropique, modèle épistatique). La canalisation d'un caractère correspond alors à l'action de sélectionner pour diminuer la sensibilité aux variations environnementales. Cette canalisation est intéressante pour répondre, par exemple, aux attentes des producteurs (optimum de production), des transformateurs (homogénéité des productions) ou aux nouvelles contraintes environnementales (agriculture durable). La mise en pratique de cette dernière passe par différentes modélisations des interactions génotype-milieu ; la plus novatrice utilise les modèles dits structuraux qui considèrent une variabilité génétique de la variance du caractère considéré et pour lesquels des développements méthodologiques sont encore nécessaires. Différentes expériences de sélection canalisante ont été mises en place sur des animaux de rente avec plus ou moins de succès. A l'heure actuelle, seule la sélection canalisante sur l'homogénéité des poids des lapereaux à la naissance est pratiquée en routine.

\section{Abstract}

\section{Canalisation and robustness: the geneticist insight}

The objective of this article was to define and illustrate the meaning of robustness in the animal genetic context where this word points to two concepts. The first one considers that robustness is the ability to maintain a global production level in stressing environments and is mainly related to the role of the corticotrop axis. The second one defines the robustness as the ability to maintain the expression of a given trait with a low variability in different environments. This concept refers to different genetic hypotheses and to the so-called «overdominance», «pleiotropic» and «epistatic» models, respectively.

The canalisation of a trait is the selection to reduce its sensitivity to environmental variations; it is an interesting strategy to reach an optimum production level, to homogenise production levels, to respond to the relaxation of environmental conditions (low input system)... Different genotype by environment interaction models can be used to canalise a trait. The most recent one is the structural 
model, which deals with the hypothesis that the expression of a trait is controlled by two sets of genes i. e. one set controlling the mean of the trait and the other set controlling the environmental variability of the trait. Several canalising selection experiments have been performed in farm animals but only canalisation for rabbit weight at birth is routinely used at the present time.

BODIN L., BOLET G., GARCIA M., GARREAU H., LARZUL C., DAVID I., 2010. Robustesse et canalisation : vision de généticiens. In : Robustesse, rusticité, flexibilité, plasticité, résilience... les nouveaux critères de qualité des animaux et des systèmes d'élevage. Sauvant D., Perez J.M. (Eds). Dossier Inra Prod. Anim., 23, 11-22. 
